\title{
Fast Wavelength Switching Lasers Using Two-Section Slotted Fabry-Pérot Structures
}

\author{
Frank Smyth, Student Member, IEEE, Eoin Connolly, Student Member, IEEE, Brendan Roycroft, Brian Corbett,
} Paul Lambkin, Member, IEEE, and Liam P. Barry, Member, IEEE

\begin{abstract}
Fast wavelength switching of a two-section slotted Fabry-Pérot laser structure is presented. The slot design enables operation at five discrete wavelength channels spaced by $10 \mathrm{~nm}$ by tuning one section of the device. These wavelengths operate with sidemode suppression ratio in excess of $35 \mathrm{~dB}$, and switching times between these channels of approximately $1 \mathrm{~ns}$ are demonstrated.
\end{abstract}

Index Terms-Laser fabrication, optical switching, semiconductor laser, switching time, wavelength tunable.

\section{INTRODUCTION}

W AVELENGTH-TUNABLE lasers are rapidly becoming a mainstream component in photonic networks. In addition to providing immediate cost savings for wavelength-division-multiplexed (WDM) networks in the areas of back-up transmitters and inventory reduction, these tunable devices may also be used for developing new network architectures in which wavelength channels can be provided where and when needed. One solution for extremely efficient bandwidth utilization in WDM networks is the use of wavelength packet routing [1], [2], in which the WDM optical packets, at different wavelengths, are generated by a fast tunable light source in conjunction with an external modulator. The wavelength packets can then be routed to specific nodes in the optical network by using simple optical filtering techniques.

Clearly a key component for implementing such networks is the wavelength tunable laser transmitter, as the overall design of the wavelength packet-switched WDM networks will be heavily dependent on the characteristics of these tunable devices. The main characteristics that determine the usefulness of wavelength tunable lasers in wavelength packet-switched systems are the wavelength tuning range, the sidemode suppression ratio (SMSR), the output power, and the speed at which the device can switch from one wavelength to another. Taking into account these characteristics, the most suitable lasers for use in wavelength packet-switched systems are electronically tunable devices [3]. Typical examples of such devices are grating-assisted codirectional coupler with sampling grating reflector (GCSR) lasers [2], [4], [5], the sample grating distributed Bragg reflector (SG-DBR) structure [6], [7],

Manuscript received April 24, 2006; revised July 5, 2006. This work was supported by Science Foundation Ireland through the Centre for Telecommunications Value Chain Driven Research.

F. Smyth, E. Connolly, and L. P. Barry are with the Research Insitute for Networks and Communications Engineering, Dublin City University, Glasnevin, Dublin 9, Ireland (e-mail: smythf@eeng.dcu.ie).

B. Roycroft, B. Corbett, and P. Lambkin are with the Tyndall National Institute, "Lee Maltings," Cork, Ireland (e-mail: brendan.roycroft@tyndall.ie).

Digital Object Identifier 10.1109/LPT.2006.882319

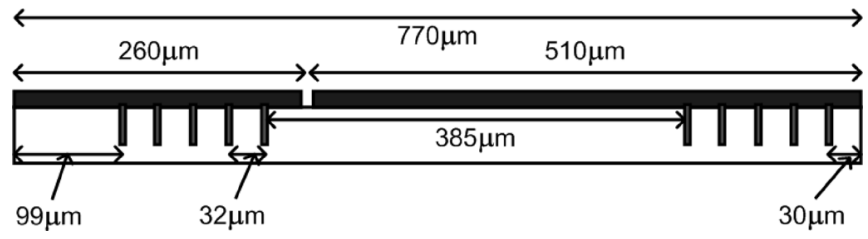

Fig. 1. Device structure of the two-section FP laser.

and super-structure grating DBR lasers [8]. These devices can achieve tuning ranges in excess of $60 \mathrm{~nm}$, SMSRs exceeding $40 \mathrm{~dB}$, output powers above $10 \mathrm{dBm}$, and switching times in the order of 5 ns using advanced electronic drive circuitry [9]. The main disadvantage of these devices is normally the complexity of fabrication, which can typically require multiple epitaxial stages that require high tolerance steps such as grating formation (as is the case for SG-DBR devices). It is thus of interest to investigate novel device structures that may be employed for the development of electronically tunable lasers.

The work carried out in this letter investigates the use of a two-section slotted Fabry-Pérot (FP) structure for developing a wavelength tunable laser diode. These devices are easier to fabricate than traditional electronically tunable devices described above as they are based on single epitaxial step. Our results show that the initial devices fabricated can discretely tune over a range of $40 \mathrm{~nm}$, and achieve an SMSR of greater than $35 \mathrm{~dB}$ for these wavelength channels. The current device design allows the laser to access channels with a spacing of $10 \mathrm{~nm}$ by variation of the current applied to one section of the slotted laser. In this work, we demonstrate the ability of such a device to switch between channels on a time scale of around $1 \mathrm{~ns}$.

\section{TunAble Laser FAbrication Using Slotted FP STRUCTURE}

To fabricate the devices used in this work, we initially fabricated conventional $3-\mu \mathrm{m}$-wide ridge waveguide lasers based on commercially available material. During the fabrication, a series of slots are introduced into the ridge that act as sites of internal reflections [10]. The slots are etched to a depth which just penetrates the top of the upper waveguide resulting in an internal reflectance of $\sim 1 \%$ at each slot. The structure of the device is shown in Fig. 1.

It has a total length of $770 \mu \mathrm{m}$ with Section 1 having a contact length of $260 \mu \mathrm{m}$ and Section 2 having a contact length of $510 \mu \mathrm{m}$. Section 1 contained five slots, spaced $32 \mu \mathrm{m}$ apart, and located $99 \mu \mathrm{m}$ from the front facet, while Section 2 contained five slots, spaced $32 \mu \mathrm{m}$ apart, and located $30 \mu \mathrm{m}$ from the back facet. 


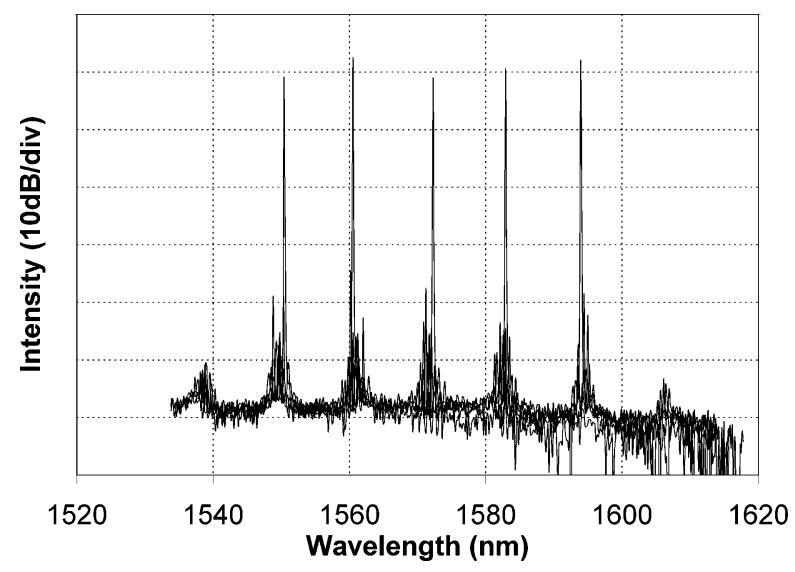

Fig. 2. Combined optical spectra from the slotted FP laser at five different operating points demonstrating single-mode operation and SMSR $>35 \mathrm{~dB}$.

Due to the relatively large reflectivity from the individual slots, a number of subcavities are formed. The 32- $\mu \mathrm{m}$ slot separation results in a free spectral range of approximately $10 \mathrm{~nm}$ which sets the supermode spacing. A second subcavity is formed between the two sets of slots and this cavity sets the dominant FP mode spacing in the laser spectrum. Finally, subcavities are formed between the slots and the uncoated facets. As the different sections of the laser are differentially driven, the local refractive index is changed due to an increase in carrier density associated with incomplete carrier clamping, a change in the local gain resulting in a change in index through the linewidth enhancement factor, and also due to current-induced heating. The latter induces a $\Delta n$ of $5 \times 10^{-5}$ per milliamp under continuous-wave conditions. These index changes result in a change in the resonant conditions favoring one of the supermodes.

\section{EXPERIMENTAL SETUP AND RESULTS}

The initial experimental analysis on the devices described above involved static characterization. Fig. 2 below shows five different optical spectra from the device overlaid on top of each other. The device has two sections, and by setting the current applied to Section 1 at $88 \mathrm{~mA}$, and varying the dc current applied to Section 2 between 80 and $140 \mathrm{~mA}$, single-mode operation with SMSR greater than $35 \mathrm{~dB}$ is available over a range of $40 \mathrm{~nm}$, with a channel spacing of approximately $10 \mathrm{~nm}$ (corresponding to the supermodes of the device). The output power of the device when coupled into fiber varied between -3 and $-7 \mathrm{dBm}$ depending on the channel selected.

As was stated above, the switching speed of a tunable laser is one of the most important features for WDM optical packet switching. In order to test the switching speed of the device, the experimental setup shown in Fig. 3 was initially used. Section 1 of the laser is driven by a constant dc current of $88 \mathrm{~mA}$, and Section 2 is driven by a square-wave signal at a frequency of $50 \mathrm{MHz}$ generated by an Anritsu pulse pattern generator. The square-wave signal switches the current applied to the second section of the laser between 120 and $140 \mathrm{~mA}$, which causes the laser to switch from 1560.5 (referred to as "Channel 1" henceforth) to $1593.9 \mathrm{~nm}$ (referred to as "Channel 2" henceforth) every $20 \mathrm{~ns}$.

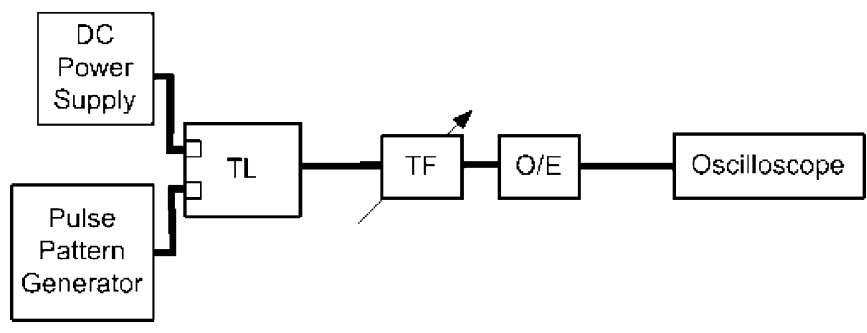

Fig. 3. Experimental configuration used to determine switching time of thelaser. Comprised of tunable laser (TL), tunable filter (TF), and photodetector $(\mathrm{O} / \mathrm{E})$.

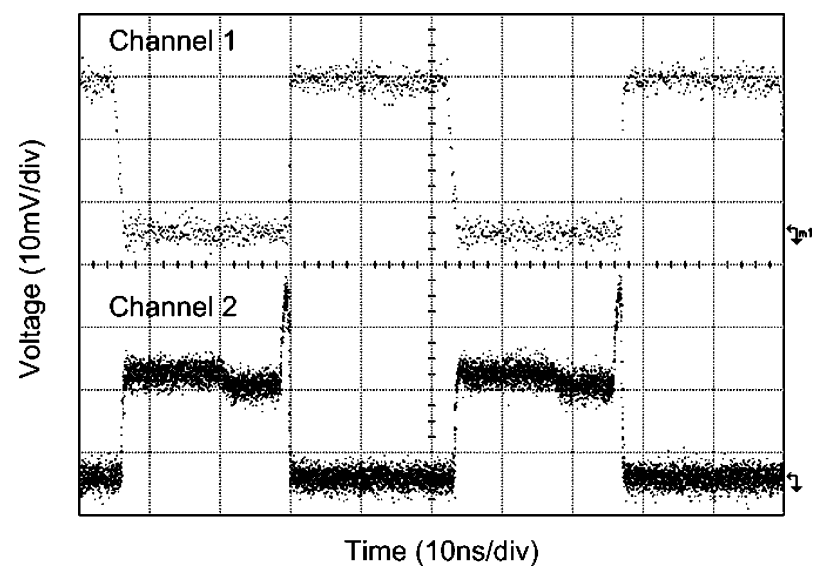

Fig. 4. Tunable laser switching between Channels 1 and 2 with the filter set on Channel 1 (top) and on Channel 2 (bottom).

By aligning an optical bandpass filter on Channel 1 and passing the output of this filter into a photodetector, we can select out that channel and view the switch on an oscilloscope. The high level in the upper waveform in Fig. 4 corresponds to the time when Channel 1 is on and hence light is passing through the filter. The low level in the upper waveform corresponds to the time that the laser is on Channel 2. The lower waveform corresponds to the case when the filter was set to select out Channel 2. From this result we can see that laser switched on a timescale of a few nanoseconds between operating wavelengths.

In order to more accurately measure how long it takes for the laser to switch wavelengths, we use a heterodyne technique similar to that employed in [11], but in our case, the beating signal is determined by setting the output frequency of an external cavity laser (ECL) to be $1 \mathrm{GHz}$ from the Channel 1 output frequency of the slotted FP laser. By placing a 1.87-GHz low-pass electrical filter after the photodetector, we can determine how long it takes the laser to get within $1.87 \mathrm{GHz}$ of the target frequency when it is switching from Channel 2 to Channel 1. Fig. 5 presents the detected electrical signal as the laser is switching between Channels 1 and 2 at a repetition rate of $50 \mathrm{MHz}$. The dc component corresponds to the time that the laser is operating on Channel 2 , and the modulated portion corresponds to the time the laser is operating on Channel 1. It should be noted that the optical output power from the laser is slightly greater on Channel 1 than on Channel 2, thus the dc level when the laser is operating on Channel 2 is less than half of the level of the modulated signal. By investigation of the time when the laser switched 

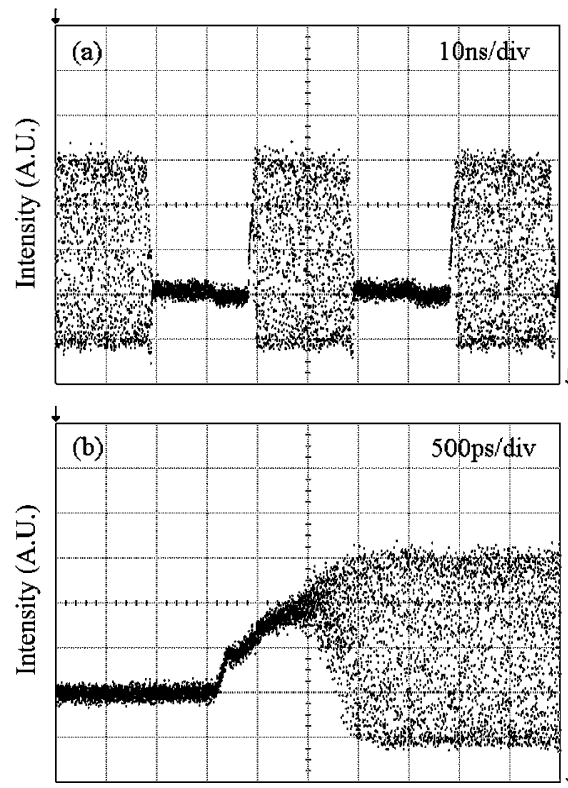

Fig. 5. Beating signal generated when output of switching laser is mixed with ECL signal $1 \mathrm{GHz}$ away from one channel from slotted laser with (a) $10 \mathrm{~ns} / \mathrm{div}$ and (b) $500 \mathrm{ps} / \mathrm{div}$.

from Channel 2 to Channel 1, we can see that the laser has completed its switch to within $1.87 \mathrm{GHz}$ of it target wavelength after about $1.3 \mathrm{~ns}$. It should be noted that unlike SG-DBR and GCSR tunable lasers that have passive tuning sections, these two-section slotted FP lasers have active sections. This ensures that the carrier lifetimes that may affect switching speed are limited by stimulated carrier lifetimes, and not spontaneous lifetimes as with SG-DBR and GCSR. It is, thus, possible to achieve very fast switching times with these devices. A similar switching time can be expected for all channel combinations due to the fact that the same tuning mechanism is responsible for each switch and we have measured the switching time for other combinations to be within $10 \%$ of the result presented here.

\section{Discussion AND CONCLUSION}

Wavelength tunable lasers are becoming a mainstream component in photonic networks. In addition to providing cost savings for WDM networks with respect to inventory reduction, these tunable devices may also be used for implementing more efficient bandwidth utilization in WDM networks by employing wavelength packet switching architectures. In this letter, we have presented a novel design of a wavelength tunable laser based on a two-section slotted FP structure. These devices are easier to fabricate than current commercial electronically tunable lasers because they are based on a single epitaxial step, and not multiple epitaxial stages that require high tolerance steps such as grating formation. Our results show that these initial devices are capable of switching between two wavelengths, to within $2 \mathrm{GHz}$ of the desired channel, on a timescale of around $1 \mathrm{~ns}$. We have also shown that they can operate with SMSRs in excess of $35 \mathrm{~dB}$ and an improvement in the SMSR can be expected by optimal design of the slot configuration and by the inclusion of phase tuning sections.

The current devices can access five wavelength channels with 10 -nm spacing by tuning the current to one section. Device modeling suggests that small spacing and higher channel counts can be achieved using the Vernier effect as defined by the slots. The channel spacing is inversely proportional to the primary distance between the slots. As an example, for 100-GHz spacing, a distance of $400 \mu \mathrm{m}$ would be required. Realization of such devices would require long waveguides with low loss. With the current devices, each wavelength channel has a continuous tuning range associated with it. As the current is varied, we have found a frequency shift of approximately $5 \mathrm{GHz} / \mathrm{mA}$ for each channel, and it is possible to vary the drive current by $10 \mathrm{~mA}$ without inducing a channel switch. This allows a minimum of $50-\mathrm{GHz}$ tuning for each channel, which allows centring on the nearest $100-\mathrm{GHz}$ ITU grid channel.

\section{REFERENCES}

[1] I. White, R. Penty, M. Webster, Y. J. Chai, A. Wonfor, and S. Shahkooh, "Wavelength switching components for future photonic networks," IEEE Commun. Mag., vol. 40, no. 9, pp. 74-81, Sep. 2002.

[2] C.-K. Chan, K. Sherman, and M. Zirngibl, "A fast 100 channel wavelength tunable transmitter for optical packet switching," IEEE Photon. Technol. Lett., vol. 13, no. 7, pp. 729-731, Jul. 2001.

[3] L. Coldren, "Monolithic tunable diode lasers," IEEE J. Sel. Topics Quantum Electron., vol. 6, no. 6, pp. 989-999, Nov./Dec. 2000.

[4] M. Kauer, M. Girault, J. Leuthold, J. Honthaas, O. Pellegri, C. Goullancourt, and M. Zirngibl, "16-channel digitally tunable external-cavity laser with nanosecond switching time," IEEE Photon. Technol. Lett., vol. 15, no. 3, pp. 371-373, Mar. 2003.

[5] P.-J. Rigole, S. Nilsson, L. Backbom, B. Stalnacke, E. Berglind, J.-P. Weber, and B. Stoltz, "Quasi-continuous tuning range from 1560 to $1520 \mathrm{~nm}$ in a GCSR laser with high power and low tuning currents," Electron. Lett., vol. 32, pp. 2352-2354, 1996.

[6] J. E. Simsarian, A. Bhardwaj, J. Gripp, K. Sherman, Y. Su, C. Webb, L. Zhang, and M. Zirngibl, "Fast switching characteristics of a widely tunable laser transmitter," IEEE Photon. Technol. Lett., vol. 15, no. 8, pp. 1038-1040, Aug. 2003.

[7] B. Mason, "Characteristics of sampled grating DBR lasers with integrated optical amplifiers," in Proc. OFC 2001, Annaheim, CA, 2001, Paper Tul6.

[8] H. Ishii, H. Tanobe, F. Kano, Y. Tohmori, Y. Kondo, and Y. Yoshikuni, "Quasicontinuous wavelength tuning in super-structure grating (SSG) DBR lasers," IEEE J. Quantum Electron., vol. 32, no. 3, pp. 433-440, Mar. 1996.

[9] J. E. Simsarian, M. C. Larson, H. E. Garrett, H. Xu, and T. A. Strand, "Less than 5-ns wavelength switching with an SG-DBR laser," IEEE Photon. Technol. Lett., vol. 18, no. 4, pp. 565-567, Feb. 15, 2006.

[10] B. Corbett and D. McDonald, "Single longitudinal mode ridge waveguide $1.3 \mu \mathrm{m}$ Fabry-Perot laser by modal perturbation," Electron. Lett., vol. 31, pp. 2181-2182, 1995.

[11] H. Joseph and D. Sadot, "A novel, self-heterodyne method for combined temporal and spectral high resolution measurement of wavelength transients in tunable lasers," IEEE Photon. Technol. Lett., vol. 16, no. 8, pp. 1921-1923, Aug. 2004. 